\title{
Interactions affecting gibberellic acid production in solid-state culture: A factorial study
}

L. M. Pastrana, M. P. Gonzalez, J. Pintado and M. A. Murado

Address reprint requests to Dr. M. A. Murado, Instituto de investigacions Mariñas

(CSIC), Eduardo Cabello, 6.3620\%Vigo, Spain

The production of GA3 in solid-state culture was studied using systems composed of maize cob particles soaked in an amylaceous efluent, as a function of three variablesparticle diameter, volume of liquid phase, and substrate concentration-with a determinant influence on the response. The empirical model obtained provides an explanation of the behavior of the system in terms of a series of interactions with underlying problems of water activity and mass transfer, that can, however, be manipulated in a simple and reproducible manner. This model also allows the suitable orientation of production improvement, the interpretation of the mechanism of substrate inhibition detected, as well as means of correcting it without reducing the initial concentration of the substrate, and leads to an 11 -fold increase in the production obtained in the preliminary assays.

Keywords: Solid-state-fermentation: wastes; GA3 production; factorial study

Introduction

In previous papers ${ }^{1-4}$ it was demons $\sim$ ated that mussel processing wastes [(MPW); average COD: $25 \mathrm{~g}_{2} \mathrm{l}^{-1}$; glycogen as main component: $10 \mathrm{~g} \mathrm{l}^{-1}$ ], an important eutrophication factor along the coast of the Galician Rias Baixas (northwest of Spain), constitute a suitable substrate for various bioproductions of potential economic interest, one of which is gibberellic acid (GA3).

Although the production of GA3 is normally carried out in submerged culture, in recent years the possibility of using solid-state culture for this purpose has attracted a great deal of attention, ${ }^{5-9}$ Based on traditional food production techniques, this resort has already extended to a growing number of industrial applications such as 
delignification, ${ }^{10-12}$ or the production of enzymes ${ }^{13-16}$ and secondary metabolites, ${ }^{17-19}$ for some of which it offers distinct advantages over the submerged culture. ${ }^{*}$

In its typical form, solid-state culture is characterized by the development of the microorganism in an environment of low water activity, upon a damp insoluble material (bran, sawdust, etc.), which functions both as a physical support and a source of nutrients. However, the fact that some of the more interesting peculiarities of this type of culture (low water activity and high oxygen transfer) do not require the coincidence of support and substrate suggests the possibility of reproducing them by means of impregnating a nutritional inert support with a nutritive solution.

It is reasonable to expect, then, that the MPW, which are suitable for the production of GA3 in submerged culture, ${ }^{4}$ are also adequate as a medium for the impregnation of a porous support, such as washed maize cob. Although the possibility of this material's contributing-as substrate, co-substrate, or enzymatic inducer-to the production of GA3 was not discarded a priori, all the results indicated the restriction of its role to that of physical support which, in any event, provides consistent responses after repeated reuse.

In the present article, we studied the variation in the productions of GA3 in this type of system from an empirical point of view, as a function of three factors-particle diameter, volume of liquid phase, and substrate concentration- which, although they cannot be considered basic state variables in the same way as, for example, water activity and oxygen transfer capacity, they enable us to understand the role of the latter in the behavior of the system and to operationally redirect the search for better responses.

Materials and Methods

General

The microorganism used was Gibberella fujikuroi (NRRL 2284). The composition of the MPW and their treatment for the preparation of the culture medium (also useful for other objectives) were described in detail in previous papers ${ }^{2-4}$; basically the preparation involves the concentration of the effluent by ultrafiltration dialysis at $100 \mathrm{~K}$ dalton, 
followed by partial hydrolysis with an amylolytic preparation obtained from cultures of Aspergillus oryzae on the raw effluent.

A typical final composition in essential nutrients of this medium (called $10 \mathrm{M}-0.5 \mathrm{H}$ ) is (in grams per liter) total sugars, 100 (reducing sugars/total sugars $=0.50$ ); total nitrogen, 0.7-0.8 (protein $\mathrm{N}, 75-90 \%$ of total $\mathrm{N}$ ); total phosphorus, 0.0550 .10 . The $\mathrm{C} / \mathrm{N}$ ratio is, therefore, in the 50-57 interval. However, because the microorganism is unable to use all of the proteic $\mathrm{N}$ present, ${ }^{4}$ the effective value is $\mathrm{C} / \mathrm{N} \sim 60$. Before autoclave sterilization (steam flow $1 \mathrm{~h}^{-1}$ ), the medium was supplemented with $\mathrm{KH}_{2} \mathrm{PO}_{4}$ to a value of $\mathrm{C} / \mathrm{P}=40$, and $\mathrm{pH}$ was adjusted to 4.0 with $2 \mathrm{~N} \mathrm{HCI}$.

The support was prepared by grinding the maize cob, followed by sieving to obtain the desired granulometry, and autoclave treatment at $121^{\circ} \mathrm{C} 15 \mathrm{~min}^{-1}$ with $\sim 10$ times its volume of water. After being filtered and washed over a Büchner funnel, the material was finally dried at $70^{\circ} \mathrm{C}$. Stored without special precautions, it was once again sterilized at $121^{\circ} \mathrm{C} 15 \mathrm{~min}^{-1}$ before use, and soaked aseptically with a suitable volume of sterile medium. When high loads of substrate were used (superior to those obtainable with the required volume of medium), two successive impregnations were carried out, with evaporation of the former at $70^{\circ} \mathrm{C}$.

The inoculum consisted of an aqueous suspension of mycelium from a 48-h submerged culture on the same medium, washed and treated for $15 \mathrm{~s}$ in an Ultraturrax. After previous calibrations (between $\mathrm{OD}_{750 \mathrm{~nm}}$ of the suitable dilutions and dry weight), the operation was adjusted in such a way that suitable volumes and weights of inoculum were obtained. The liquid phase in the culture was defined as the sum of the volumes of medium and inoculum. The incubations were carried out in duplicate in a $150-\mathrm{ml}$ Erlenmeyer flask at $30^{\circ} \mathrm{C}$, in a closed water bath and in static conditions, with a load of $5 \mathrm{~g}$ of support by Erlenmeyer flask. To avoid possible agglomerations of the material due to growth of the microorganism, the cultures were manually shaken once a day.

Analytical methods

At preestablished times, the materials of each flask were macerated for $1 \mathrm{~h}$ in distilled water with orbital shaking at $100 \mathrm{cpm}$; the support was separated by filtration $(100-\mu \mathrm{m}$ 
nylon mesh); and the extract was centrifuged. The sediment, washed with distilled water and dried at reduced pressure on $\mathrm{P}_{2} \mathrm{O}_{5}$ at $55^{\circ} \mathrm{C}$ could be used for approximate estimation of the biomass. In the supernatant, total carbohydrates, proteins, nitrogen, phosphorous, and gibberellic acid were determined. The corresponding analytical methods, as well as that applied to reducing sugars, were described or referred to in previous papers. ${ }^{1,3}$

\section{Experimental designs}

To obtain the empirical equation that describes the production of GA3, a complete firstorder orthogonal design ${ }^{21,22}$ was used, with quadruple replication in the center of the domain. The subsequent extension of the experimental domain was guided by the linear approximation gradient method, taking into account experimental reasonableness, as indicated sufficiently in the discussion of results.

Results and Discussion

Preliminary evaluation of the system

To establish the basic characteristics of the system, in a first assay the four series of cultures obtained by combining two different liquid phases (distilled water and medium $10 \mathrm{M}-0.5 \mathrm{H}$ ) with two types of support (untreated and exhaustively washed cob) were studied. In all cases the load per experimental unit was $5 \mathrm{~g}$ of support (diameter $=4$ $\mathrm{mm})$ and a $7-\mathrm{ml}$ liquid phase, which included $5 \mathrm{ml}$ of medium $\left(100 \mathrm{mg} \mathrm{ml}^{-1}\right.$ of carbohydrates) and $2 \mathrm{ml}$ of an inoculum containing $20 \mathrm{mg}$ (dry weight) of mycelium.

The results indicated that the use of the untreated support totally suppressed not only the production of gibberellins, but also that of bikaverins, the pigments responsible for the conspicuous red coloring detected, in cultures on washed support, after $\sim 3$ days of incubation, indicating the beginning of limitation by nitrogen. It can be said, therefore, that the support contained $\mathrm{N}$ in sufficient proportion to prevent the limitation necessary for the synthesis of the hormone, and contained it in a form that could be eliminated by the washing procedure applied. Furthermore, the production of the biomass in the four types of culture decreased in the order: untreated $M>$ washed $M>$ untreated water $>$ washed water, with the recovery in the latter case of a slightly inferior biomass to that of 
the inoculum, which suggests the scant (or null) nutritive value of the washed support for the microorganism.

Figure 1 compares the results corresponding to the only situation (washed M) capable of promoting the production of the hormone with those obtained in the same conditions, but with a double concentration of substrate (200 $\mathrm{mg} \mathrm{g}^{-1}$ of support). The productions of GA3 indicate regular tendencies throughout the incubation period and representative values from $200 \mathrm{~h}$ on. The fact that not only productivity with regard to the substrate consumed, but also absolute production of the hormone was inferior in the presence of the high level of carbohydrates was the most noteworthy aspect, and suggests a substrate inhibition process similar to that indicate by Kumar and Lonsane. ${ }^{7}$ The level of stabilization of the consumption of $\mathrm{N}$, slightly higher in the more concentrated medium, repeated the behavior already discussed ${ }^{4}$ in submerged cultures of the same species on the same type of media.

Finally, neither in the consumption of carbohydrates nor in the production of GA3 obtained in this last experimental situation were any significant differences detected in five successive reuses of the same support.

Interactions affecting GA3 production in the system studied

To obtain a quantitative description of the effects of different factors of the system under investigation likely to be involved in the production of the hormone, a complete 2 factorial plan was carried out with three variables (D: diameter of the support, L: liquid phase in the system, and S: concentration of carbohydrates) whose domains and codification are indicated in Table 1. Table 2 shows the experimental matrix, as well as the corresponding production of GA3, an analysis of the significance of the model proposed, and the results related to carbohydrate consumption. With Y as the production of GA3 (in micrograms per gram of support) after a 200 -h incubation period, equation obtained.

$$
\begin{gathered}
\mathrm{Y}=428.2-53.9 \mathrm{D}+301.6 \mathrm{~L}-89.1 \mathrm{DL} \\
+ \text { 70.13 DS + 65.4 DLS }
\end{gathered}
$$

is highly consistent. Thereby, with the acceptable coefficients according to the Student $\mathrm{t}$ test with $\alpha=0.05$, model (1) is significant when applying the Fisher $\mathrm{F}$ test (with $\alpha=$ 
0.05 , and even when extending the rejection region to $\alpha=0.1$ ) to the total error/experimental error ratio as well as to lack of fitting/experimental error ratio. Raising the requirements of the $t$ test to $\alpha=0.02$, the model maintains significance at the strictest level in the F tests without losing any terms. Also, none of the rejected terms are acceptable even when relaxing the requirements of the $\mathrm{f}$ test to $\alpha=0.1$.

Moreover, there is a remarkably high correlation between observations and predictions, plotted in Figure 3, which also shows the standardized residues, whose maximum values are situated near $1 \mathrm{SD}$ and whose grouping will be discussed subsequently.

Given the relative magnitudes' of the coefficients, it can be concluded, first of all, that the system is highly susceptible to the factors studied, as the response in the center of the domain (independent term) can be raised to more than twice its value within the experimental region. Moreover, it seems quite clear that, despite the importance of the first order effect, particularly of the liquid phase, the interactions play an essential role not only from a quantitative point of view, but also as a means of explaining the behavior of the system in terms of the more characteristics structural factors of the culture in solid state-that is, water activity and oxygen transfer, with the latter being considerably important in the production of GA3.

In fact, when the three terms that involved the variable $L$ are considered jointly, apart from the strongly positive first order effect observable in any situation (Figure 2), it can be said that the negative sign of the DL interaction suggests that when the interparticular space increases on increasing the diameter of the particle, a high liquid phase contributes to an excessive rise of water activity, making the culture drift toward a poorly oxygenated (given its scant shaking) submerged, and therefore less productive, condition. However, the positive sign of the DLS interaction indicates that the previous effect is antagonized when high concentrations of substrate generate excess biomass capable of absorbing sufficient water in the interparticular space. Although in this instance the biomasses were not determined, the relationship between the initial level of carbohydrates and the biomasses found in the preliminary assay (Figure 1), along with the consumptions of carbohydrates detected (Table 2), leaves little doubt in this respect. 
It is to be expected, then, that with low diameters (small interparticular space) the increase of the substrate would exert an opposite effect, provoking a decrease in production due to the limitations in oxygen transfer determined by the excessive compactness of the system. This is exactly what occurs, as can be appreciated when comparing the maximum points of both parts of Figure 2. Moreover, the fact that this effect disappears when the liquid phase is low (minimum points of both parts of Figure 2) suggests an additional action, under these circumstances, of a more drastic limitation, probably the conjunction of the decrease of the liquid phase and the increase in the available absorbing surface (decrease of diameter), which determines a suboptimum level of water activity.

Finally, the initially surprising absence, of the $\mathrm{S}$ variable between the first-order terms can be justified by observing its direct contribution to contrasting effects, which later cancel themselves out (production of biomass, antagonism of excess of $\mathrm{L}$, and compactness); therefore, its influence only results in interactions whose global effect is negative, except at high simultaneous values of D and L. Nevertheless, the distribution of the residues shown in Figure 3 demonstrates that, independently of the values of the rest of the variables, the responses to high $\mathrm{S}$ are lower than expected, but in instances of low $\mathrm{S}$ the contrary occurs. It is reasonable to suppose that this suspicious regularity reflects a negative effect characteristic of the $\mathrm{S}$ variable (substrate inhibition?), which does not manage to incorporate itself in the model because of its low entity (particularly in the series with $L=-1$ ) in relation to the variance of experimental error.

Extrapolation of the model

A useful criterion in attempting to improve the response beyond the experimental domain consists of following the linear approximation gradient (LAG) of the model obtained. Differentiating the expression (1) with respect to each of the independent variables $\left(\mathrm{X}_{\mathrm{i}}\right)$ :

$$
\begin{aligned}
& \partial Y / \partial D=-53.9-89.1 L+70.13 S+65.4 L S \\
& \partial Y / \partial L=301.6-89.1 D+65.4 D S \\
& \partial Y / \partial S=70.13 D+65.4 D L
\end{aligned}
$$

and substituting the $X_{i}$ in the resulting expressions (2) to (4) with the values corresponding to any point of the domain, the slopes of the function in this point are 
obtained, in the direction of each variable. If the first point of the LAG is now established in the center of the domain $\left(\mathrm{X}_{\mathrm{oi}}=0\right)$, the coordinates $\left(\mathrm{X}_{\mathrm{ij}}\right)$ of the following point can be calculated, once arbitrary increments $\Delta \mathrm{X}$, have been assigned to the independent variables, by means of:

$$
X_{1 i}=X_{o i}+\left(\partial Y / \partial X_{i}\right) \cdot \Delta X_{i}
$$

with the corresponding response being the value that is obtained by substituting in (1) the $\mathrm{X}_{\mathrm{i}}$ for the values $\mathrm{X}_{1 \mathrm{i}}$. From the point $\mathrm{X}_{\mathrm{ji}}$ the $\mathrm{X}_{2 \mathrm{i}}$ would then be calculated by repeating the procedure, and doing so successively.

The trajectory of the LAG thus determined depends not only on the starting model, but also on the increments $\Delta X_{i}$, which should therefore be fixed using criteria experimentally reasonable. Proceeding in this way, the trajectory denoted as A in Figure 4 (and which implies the following domains, in coded values: D: 0 to 3.5 ; L: to 2.5 ; S: 0 to -1.1) could constitute a good extrapolation criterion, with interesting expected responses.

Nevertheless, if the expected values deviated perceptibly with regard to those observed, it could be difficult to identify the variables responsible for the lack of fit when all of them varied simultaneously. This led to a simpler extrapolation strategy, in which only the diameter varied $(-1.00,-1.75,-2.40 \mathrm{y}-2.90$ in coded values), and the two remaining variables were fixed at the values $\mathrm{L}=2$ and $\mathrm{S}=-0.8$ which, according to the analysis of the previous section, favor the response and seem to be sufficiently realistic. In this second option, the expected responses are found on trajectory B of Figure 4.

Up to the point in which $\mathrm{D}=-2.40$, the experimental results obtained show a noteworthy adjustment with the extrapolation given by trajectory B and, as expected, given the values of the variables $\mathrm{L}$ and $\mathrm{S}$ in the corresponding cases, trajectory $\mathrm{A}$ predicts productions with errors by defect that are greater the nearer they are to the already tested experimental domain. From that value, the real production is perceptibly better than that expected. Although this is very satisfactory from a practical point of view, it requires an attempt at explanation. 
A plausible approach to an explanation could lie in the nature of the interactions. In fact, the increase of the response due to the growing values of $\mathrm{L}$ and decreasing values of $\mathrm{D}$ not only depends on the first-order terms, but also on the interactions of these two variables between themselves and with the substrate. Moreover, the analysis of the system proposed in the previous section makes it reasonable to suppose that the decrease of D (increase of particle surface) allows, without increasing the free water, an increase of $\mathrm{L}$ in more than linear manner, which would mean that even if its variables were accepted, model (1) would underestimate some of the interactions if applied to an extrapolation area. In support of this point of view is the fact that when doubling the coefficient of the DLS term, the linear approximation gradient generates a trajectory (C in Figure 4) which, in the area furthest away from the first domain, adjusts to the experimental results better than any of the previous ones.

Despite the interest of the last finding and the fact that the extrapolation results suggest still increasing production possibilities, it nevertheless seems clear that the increase in response due to the DLS term will eventually clash with the restrictions characteristic of the system that are already noticeable in the first domain-that is, a drift toward submerged culture on the one hand, and the difficulties in oxygen diffusion caused by an excess compactness on the other.

Effects of the substrate

The upper part of Figure 5 shows the time course of three cultures carried out again at D $=-2.90$ and $\mathrm{L}=2$ (coded values), in the presence of three concentrations of substrate $(\mathrm{S}$ $=-1, \mathrm{~S}=\mathrm{O}$ y $\mathrm{S}=2$; or, 50, 100, and $200 \mathrm{mg} \mathrm{g}^{-1}$ of support). The productions obtained, coherent with the revious results, reached a maximum of $3.8 \mathrm{mg} \mathrm{GA}^{-1} \mathrm{~g}^{-1}$ of support and revealed, when comparing them to those of the preliminary trial (Figure 1), the noteworthy effect of the variables $\mathrm{D}$ and $\mathrm{L}$, whose suitable modification led to multiplying the production of GA3 at $200 \mathrm{~h}$ of incubation by a factor of about 11 .

Nevertheless, the most remarkable aspect of this last series of cultures was that, although the decrease in yield was clearly manifested when raising the initial level of the substrate, the negative effect of this variable observed in Figure 1 did not appear in this case. This confirms the analysis whereby the effect of the substrate cannot be 
separated from the remaining variables of the system, and poses a question concerning the structural causes of substrate inhibition.

In fact, the usual definition of substrate inhibition (SI) is purely formal. It is said that a response (i.e., the production of GA3 in a determined period) is affected by SI when the increase of the concentration of the substrate promotes the increase of the production only to a maximum, from which further elevations determine the decrease of this production (when the latter increases asymptotically with the concentration of the substrate, this is merely a case of decreasing yields). If, according to the Monod model, one accepts the application of the Michaelis-Menten equation to the productions of microbial cultures, one can also accept the formal description of SI by means of the equation:

$V=\frac{V_{m} S}{K_{m}+S+K_{i} S^{2}}$

with variables $\mathrm{V}$ (production rate) and $\mathrm{S}$ (substrate concentration), and with the parameters $\mathrm{V}_{\mathrm{m}}$ (maximum production rate $\left(, \mathrm{K}_{\mathrm{m}}\right.$ (Michaelis-Menten or Monod constant), and $\mathrm{K}_{\mathrm{j}}$ (substrate inhibition constant). Nevertheless, it should be noted that, in contrast to what occurs in inhibitions by other agents (competitive, acompetitive, and noncompetitive), whose mechanism is clearly specified, SI can respond to a great variety of causes, ranging from those linked with steric hindrances or mass transfer restrictions ${ }^{23}$ to the accumulation of metabolites.

Nonetheless, if in the two last series of cultures studied here the productions of GA3 at $200 \mathrm{~h}$ corresponding to identical conditions in the variables D and L (lower part of Figure 5) are compared, it can undoubtedly be concluded that an SI process exists. Furthermore, although the adjustment of the experimental values to Equation (5) should be understood basically as a qualitative illustration (continuous trace curve in Figure 5), it is true that this adjustment generates reasonable parametric values. Kumar and Lonsane $^{7}$ reach a similar conclusion after obtaining the productions also represented at an appropriate scale in Figure 5, with another strain of G. fujikuroi in solid state on a system of wheat bran supplemented with starch.

Therefore, there seems to be no problem in affirming that the production of GA3 in solid state is affected by an SI process. But such an affirmation, although formally 
correct, does not necessarily imply that the variations in the concentrations of the substrate constitute the only determining factor in such an inhibition process. The behavior of the system, reflected as much by the empirical model (1) as by subsequent results, strongly suggests that the mechanism underlying SI here consists of a restriction to the mass transfer (to the diffusion of oxygen), determined by the relative proportions of the biomass and/or free water in the interparticular space, in turn a function of the diameter of the particles.

Consequently, in the same way as in the apparent SI detectable in the hydrolysis of starch by glucoamylase, the increase in agitation contributes to the decrease of the effect $^{23}$; in this case, the variation of other factors (liquid phase and particle diameter) also constitute means that counteract the SI in a more efficient and less damaging way for the absolute production of GA3 than the actual variation in the concentration of the substrate.

Fed-batch culture

Fed-batch operations are the more commonly recommended means to correct the effect of SI, as well as the most frequently applied to this end. In this case, the evaluation of this resort was carried out, maintaining the values (codified) $\mathrm{D}=-2.90$ and $\mathrm{L}=2$, through the comparison of the time course of the production of GA3 in the following two series of culture:

A. Initial concentration of substrate: $50 \mathrm{mg} \mathrm{g}^{-1}$ of support. Feeding at incubation times $7,11,18$, and 25 days, with medium volumes necessary to reach, after the final addition, a contribution of substrate of $200 \mathrm{mg} \mathrm{g}^{-1}$ of support.

B. Initial concentration of substrate: $200 \mathrm{mg} \mathrm{g}^{-1}$ of support. Feeding as in series A, but substituting the medium with distilled water.

The corresponding results (Figure 6) indicated that, although during the first $300 \mathrm{~h}$ of incubation, absolute production did not show significant differences in either series, and was even greater in the conventional culture, from this moment on they were overtaken by the fed-batch culture which at its maximum $\left(4.8 \mathrm{mg} \mathrm{g}^{-1}\right.$ of support) exceeded the other series by $14 \%$. On the other hand, the yield with regard to the carbohydrate 
consumption was superior in the fed-batch culture throughout the whole incubation period.

The remission in the depressor effect of a high initial concentration of substrate thus obtained enabled us again to affirm the correction of an SI problem. Yet again, it is also reasonable to suppose that, in view of the evolution of the consumptions of carbohydrates, this was due to the greater proportions of biomass in the interparticular space that are to be expected in the conventional culture-that is, that a problem of mass transfer is involved.

Moreover, when the correlations between carbohydrate consumptions and GA3 productions are examined in Figure 6, it becomes evident that the difference between both types of culture basically results in the fact that the production of the hormone progresses in a more gradual manner in the fed-batch. This suggest a contrast between a feeding procedure that leads to a conventional idiophase, and another that provokes the extension of an early idiophase (or even a series of idiophases). Therefore, and perhaps more than of SI, one should talk of control of the metabolic state of the microorganism (or even the correction of a simple process of decreasing yields).

Acknowledgments

The authors thank CICYT and Xunta de Galicia for their financial support (projects ALI 789-90, AMB 93-0 136, and XUGA-40204B-92).

References

1. Gonzalez, M. P., Siso, M. I. G., Murado, M. A., Pastrana, L. Montemayor, M. I., and Miron, J. Depuration and valuation of mussel-processing wastes. Characterization of amylolytic postincubates from different species grown on an effluent. Biores. Technol. 1992, 42, 133-140

2. Murado, M. A., Siso, M. 1. G., Gonzalez. M. P., Montemayor, M. I., Pastrana, L. and Pintado, J. Characterization of microbial biomasses and amylolytic preparations obtained from mussel processing waste treatment. Biores. Technol. 1993, 43, 117125 
3. Murado, M. A., Gonzalez, M. P., Pastrana, L., Siso, M. I. G., Miron, J. and Montemayor, M. I. Enhancement of the bioproduction potential of an amylaceous effluent. Biores. Technol. 1993, 44, 155-163

4. Pastrana, L., Gonzalez, M. P., and Murado, M. A. Production of gibberellic acid from mussel processing wastes in submerged batch culture. Biores. Technol. 1993, 45, 213-221

5. Prapulla. S. G.. Thakur. M. S. Prema, P. Lonsane. B. K. Ravindranath, B., Ramakrishna. S. V. and Sreenivasa Murthy, V. Studies on recovery, purification and estimation of gibberellic acid produced by solid state fermentation. Third Indian Convention of Food Scientists and Technologist. Mysore. 1983, 88

6. Kumar. P. K. R. and Lonsane, B. K. Gibberellic acid by sold state fermentation: Consistent and improved yields. Biotechnol. Bioeng. 1987, 30, 267-271

7. Kumar. P. K. R. and Lonsane. B. K. Potential of fed-batch culture in solid state fermentation for production of gibberellic acid. Biotechnol. Len. 1987. 9, 179-182

8. Kumar. P. K. R. and Lonsane, B. K. Microbial production of gibberellins: State of the art. Adv. Appl. Microbial. 1989. 28, 30-140

9. Kumar. P. K. R. and Lonsane, B. K. Solid state fermentation: Physical and nutritional factors influencing gibberellic acid production. Appl. Microbial. Biotechnol. 1990, 34, 145-148

10. Abdullah. A. L., Tengerdy, R. P. and Murphy, V. G. Optimization of solid substrate fermentation of wheat straw. Biotechnol. Bioeng. 1985, 21, 20-27

11. Agosin, E., Jarpa. S. Rojas. E. and Espejo, E. Solid state fermentation of pine sawdust by selected brown-rot fungi. Enzyme Microb. Technol. 1989, 11, 511-517

12. Reid, I. D. Optimization of solid-state fermentation for selective delignification of aspen wood with Phlebia tremellosa. Enzyne Micrab. Technol. 1989, 11, 804-809

13. Vallander. L. and Eriksson, K. E. Enzymic saccharification of pretreated wheat straw. Biotechnol. Bioeng. 1985, 27, 650-659

14. Desgranges. C. and Durand. A. Effect of $\mathrm{pCO}_{2}$ on growth. conidiation, and enzyme production in solid-state culture on Aspergillus niger and Trichoderma viride TS. Enzyme Microb. Technol. 1990, 12, 546-55 I

15. Rajaram. S. and Varma, A. Production and characterization of xylanase from Bacillus thermoalkalophilus grown on agricultural wastes. Appl. Microbial. Biotechnol. 1990, 34, 141-144 
16. Ramesh. M. V. and Lonsane. B. K. Critical importance of moisture content of the medium in alpha-amylase production by Bacillus licheniformis M27 in a solid-state fermentation system. Appl. Microbiol. Biotechnol. 1990. 33, 501-505

17. Lindenfelser. L. A. and Ciegler, A. Solid-substrate fermentor for ochratoxin A production. Appl. Microbial. 1975, 29, 323-327

18. Hesseltine, C. W. Solid state fermentations. Biotechnol. Bioeng. 1972, 14, 517-532

19. Hesseltine, C. W. Solid state fermentation. Part 1. Process Biothem. 1917. 12. 24-27

20. Aidoo, K. E.. Hendry. R. and Wood, B. J. B. Solid substrate fermentations. Adv. Appl. Microbial. 1982, 28, 201-238

21. Akhnazarova, S. and Kafarov, V. Experiment Optimization in Chemistry, and Chemical Engineering Moscow, MIR Publishers. 1982

22. Box, G. E. P., Hunter, W. G. and Hunter, I. S. In (Reverté ed.). Estadistica para investigadores, Barcelona, 1989

23. Miranda, M., Murado, M. A. Sanroman, A. and Lema, J. M. Mass transfer control of enzymatic hydrolysis of polysaccharides by glucoamylase. Enzyme Microb.

Technol. 1991, 13, 142-147 
Figure 1 Growth of Gibberella fujikuroi and GA3 production on the system maize cob and 10M-0.5H medium (preliminary assay), 100 (open symbols) and 200 (closed symbols) mg of total sugars per gram of support. B: Biomass, TS: total sugars; TSc: total sugar consumption; $\mathrm{N}$ : total nitrogen. Mean of two culture, two analytical replications $(2.8 \%<\mathrm{SE}<3.3 \%)$

Figure 2 Response surfaces corresponding to the GA3 production as a function of particle diameter and liquid phase, at two values of initial concentration of substrate (S) after $200 \mathrm{~h}$ incubation, independent variables are in coded values

Figure 3 (Top) Correlation between observed and expected values according to the model proposed. (Bottom) Standardized residuals corresponding to the experimental matrix arranged according to increasing response values. The arrows indicate regularities attributable to the effect of the substrate (see text). Notation of the variables are as in Table 1

Figure 4 Productions of GA3 (closed symbols) obtained in the extension of the experimental domain, represented as a function of diameter with $\mathrm{L}=2$ and $\mathrm{S}=-0.8$. Lines $\mathrm{A}, \mathrm{B}$, and $\mathrm{C}$ correspond to the expected values according to three different extrapolation criteria (see text)

Figure 5 (Top) Time course two cultures carried out at $\mathrm{D}=-2.90$ and $\mathrm{L}=2$ (coded values), in the presence of three concentrations of substrate ( $\nabla 50, \diamond 100$, and $\circ 200 \mathrm{mg}$ per gram of support). (Bottom) Production of GA3 as a function of the concentration of the substrate. $\circ$ : This work, $200 \mathrm{~h}$; $\bullet$ and $\bullet$ : according to Kumar and Lonsane, ${ }^{7}$ at 144 and $168 \mathrm{~h}$, respectively. The solid line represents an illustration of the adjustment of the experimental values ( $($ ) to the Michaelis-Menten model with substrate inhibition

Figure 6 Comparison of the results of conventional batch $(\square)$ and fed-batch $(\circ)$ cultures, with $\mathrm{D}=-2.90$ and $\mathrm{L}=2$ (coded values), according to the specifications of the text. TSc: Total sugar consumption

Table 1 Experimental domain and codification of variables used in the fractorial plan 


\begin{tabular}{|c|c|c|c|c|c|c|c|c|c|c|}
\hline \multirow{2}{*}{\multicolumn{2}{|c|}{ Coded values }} & \multicolumn{7}{|c|}{ Natural values } & & \\
\hline & & \multicolumn{2}{|c|}{$\begin{array}{l}\text { Diameter D } \\
(\mathrm{mm})\end{array}$} & \multicolumn{3}{|c|}{$\begin{array}{l}\text { Liquid Phase L } \\
\left(\mathrm{ml} \mathrm{g}^{-1}\right)\end{array}$} & \multicolumn{2}{|c|}{$\begin{array}{l}\text { Substrate } \mathrm{S} \\
\left(\mathrm{mg} \mathrm{g}^{-1}\right)\end{array}$} & & \\
\hline \multicolumn{2}{|l|}{-1} & \multicolumn{2}{|c|}{2.5} & \multicolumn{3}{|c|}{0.6} & \multicolumn{2}{|l|}{50} & & \\
\hline \multicolumn{2}{|l|}{0} & \multicolumn{2}{|c|}{3.5} & \multicolumn{3}{|c|}{1.2} & \multicolumn{2}{|l|}{100} & & \\
\hline 1 & & \multicolumn{2}{|c|}{4.5} & \multicolumn{3}{|c|}{1.8} & \multicolumn{2}{|l|}{150} & & \\
\hline \multirow{2}{*}{\multicolumn{11}{|c|}{$\begin{array}{l}\text { Codification: } \mathrm{V}_{\mathrm{c}}=\left(\mathrm{V}_{\mathrm{n}}-\mathrm{V}_{\mathrm{o}}\right) / \Delta \mathrm{V}_{\mathrm{n}} \text {; decodification: } \mathrm{V}_{\mathrm{n}}=\mathrm{V}_{\mathrm{o}}+\left(\Delta \mathrm{V}_{\mathrm{n}} \cdot \mathrm{V}_{\mathrm{c}}\right) . \mathrm{V}_{\mathrm{c}} \text { : coded } \\
\text { value; } \mathrm{V}_{\mathrm{n}} \text { : natural value; } \mathrm{V}_{\mathrm{o}} \text { : natural value in the center of the experimental domain; } \\
\Delta \mathrm{V}_{\mathrm{n}} \text { : increment of } \mathrm{V}_{\mathrm{n}} \text { corresponding to one unit of } \mathrm{V}_{\mathrm{c}}\end{array}$}} \\
\hline & & & & & & & & & & \\
\hline $\mathrm{D}$ & $\mathrm{L}$ & $\mathrm{S}$ & $\mathrm{Y}$ & $\hat{\mathrm{Y}}$ & \multirow{2}{*}{$\begin{array}{l}\text { Coefs } \\
428.2\end{array}$} & te & \multicolumn{2}{|l|}{ Model } & \multirow{2}{*}{$\begin{array}{l}\text { TsC } \\
40.8\end{array}$} & $(\%)$ \\
\hline-1 & -1 & -1 & 97 & 96 & & 80.1 & 428.2 & & & $(81.5)$ \\
\hline 1 & -1 & -1 & 158 & 157 & -53.9 & 8.2 & -53.9 & $\mathrm{D}$ & 43.6 & $(87.2)$ \\
\hline-1 & 1 & -1 & 1027 & 1008 & 301.6 & 46.1 & 301.6 & $\mathrm{~L}$ & 40.2 & $(80.3)$ \\
\hline 1 & 1 & -1 & 470 & 451 & -9.6 & 1.5 & NS & $\mathrm{S}$ & 42.4 & $(84.8)$ \\
\hline-1 & -1 & 1 & 86 & 87 & -89.1 & 13.6 & -89.1 & DL & 125.3 & $(83.5)$ \\
\hline 1 & -1 & 1 & 166 & 167 & 70.1 & 10.7 & 70.1 & DS & 136.2 & $(90.8)$ \\
\hline-1 & 1 & 1 & 719 & 737 & -8.9 & 1.4 & NS & LS & 137.7 & $(91.8)$ \\
\hline 1 & 1 & 1 & 704 & 722 & 65.4 & 9.9 & 65.4 & DLS & 140.5 & (93.7) \\
\hline 0 & 0 & 0 & 448 & 428 & & & & & & \\
\hline 0 & 0 & 0 & 437 & 428 & & & & & & \\
\hline 0 & 0 & 0 & 420 & 428 & & & & & & \\
\hline 0 & 0 & 0 & 406 & 428 & & & & & & \\
\hline
\end{tabular}

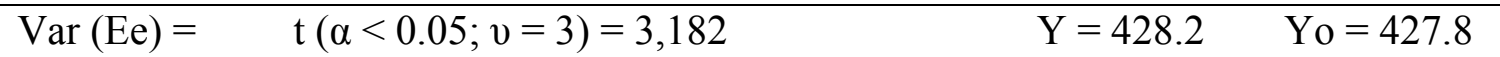
342.9

\begin{tabular}{llll}
\hline & SS & $v$ & QM \\
\cline { 2 - 4 } Model & 888118.6 & 5 & 177623.7 \\
Error & 2401.1 & 6 & 400.2 \\
Experimental & 1028.8 & 3 & 342.9 \\
error (Ee) & & & \\
\hline
\end{tabular}




\begin{tabular}{|c|c|c|c|}
\hline $\begin{array}{l}\text { Lack of fit } \\
\text { (LF) }\end{array}$ & 1372.3 & 3 & 457.4 \\
\hline \multirow{3}{*}{$\begin{array}{l}\text { Total } \\
\mathrm{r}^{2}=0.997\end{array}$} & 890519.7 & 11 & 89056.3 \\
\hline & adj. $r^{2}=$ & & \\
\hline & 0.995 & & \\
\hline \multirow{3}{*}{$\begin{array}{l}\text { QME/QMEe } \\
=1.167\end{array}$} & $F_{3}^{6}(\alpha=$ & & \\
\hline & $0.05)=$ & & \\
\hline & 8.940 & & \\
\hline \multirow{3}{*}{$\begin{array}{l}\text { QMLF/QMEe } \\
=1.334\end{array}$} & $F_{3}^{3}(\alpha=$ & & \\
\hline & $0.05)=$ & & \\
\hline & 9.280 & & \\
\hline
\end{tabular}

Y: GA3 ( $\left.\mu \mathrm{g} \mathrm{g}^{-1}\right)$; TSc: total sugars consumption ( $\mathrm{mg} \mathrm{g}^{-1}$ and \%); NS: not significant coefficient; SS: sum of squares; QME, QMEe. and QMLF: quadratic means of the total error, experimental error, and lack of fit, respectively. Variables according to Table 1 